\title{
Cataloguing stiffness anisotropy of natural sedimentary soils - From clays to intermediate soils
}

\author{
Satoshi Nishimura ${ }^{\text {i) }}$ and Delvin Abdiel Martínez ${ }^{\text {ii) }}$
}

i) Associate professor, Faculty of Engineering, Hokkaido University, Kita-13 Nishi-8, Kita-Ku, Sapporo, 060-8628, Japan. ii) Formerly graduate student, Graduate School of Engineering, Hokkaido University, Kita-13 Nishi-8, Kita-Ku, Sapporo, 060-8628, Japan.

\begin{abstract}
Natural sedimentary soils often exhibit marked anisotropy from early stages of deformation, in some cases manifesting even a few times greater stiffness in one direction than in another. Capturing this characteristic will lead to more accurate prediction and rational interpretation of ground movements in $2 \mathrm{D}$ and $3 \mathrm{D}$ problems. However, rigorous assessment of the 'effective-stress-based' stiffness anisotropy in laboratory tests requires considerable skill and expertise, particularly for fine-grained soils, which require slow, stable loading and measuring capabilities allowing full drainage. This study set out to catalogue and demonstrate general typologies of small-strain anisotropy found in naturally sedimented fine-grained soils to offer geotechnical designers a rough guide to stiffness estimation. A mechanical framework and experimental/interpretative innovations to facilitate the above are summarised.
\end{abstract}

Keywords: sedimentary soils, stiffness anisotropy, soil characterisation

\section{INTRODUCTION}

Soils, cemented or uncemented, generally exhibit elastic behaviour under small deformation $(<0.001 \%$ strain) after a sufficient creep. Although a plastic strain gradually appears beyond this fairly small threshold level, the anisotropy, in many case axi-symmetric with regard to the vertical (i.e. cross-anisotropic) in nature, observed for the initial small-strain of stiffness can have lingering effects on ground deformation patterns, and in some cases, ground stability as well. In addition to its obvious relevance to deformation problems in practice (i.e. Simpson et al, 1996; Addenbrooke et al., 1997), the first author (Nishimura, 2006), in his study on heavily over-consolidated high-plasticity London Clay, pointed out its potential relevance to stability problems too. High-OCR, high-plasticity clays tend to fail in brittle manners when hitting a bounding surface, without a clear tendency to dilate towards the critical state. Initial stiffness anisotropy leads to a significant effective stress path inclination $\left(\left(\sigma_{v}-\sigma_{h}\right) / p^{\prime}\right.$; see also Graham and Houlsby, 1983), resulting in a marked undrained shear strength anisotropy even when anisotropy of the effective stress-based failure envelope is not equally significant, as illustrated in Fig. 1.

The engineering importance of understanding small-strain anisotropy is clear, but it is often missed out in geotechnical designs or ground movement interpretations. Among many reasons for this, including an inevitable need for simplification in many situations and technical difficulties involved in experimentally quantifying each modulus, the authors suspect insufficient general recognition of the severity of small-strain stiffness anisotropy and its distinct typologies encountered in fine-grained soils. A typical example of this is taking $E_{v}{ }^{u}=3 G_{v h} \quad\left(E_{v}{ }^{u}\right.$ : vertical undrained Young's modulus and $G_{v h}$ : shear modulus for deformation within vertical planes) as evidence of isotropy. This paper will demonstrate that this leads to very erroneous assessment of stiffness characteristics in many soils.

Numerous studies have presented stiffness moduli against different deformation modes and offered partial views of the stiffness anisotropy. However, a method to experimentally measure, parameterise and present it in a fully multi-dimensional manner has not been established, let alone standardised. Nishimura (2014a) adopted an innovative approach originally proposed by Kuwano et al. (2000) and Lings et al. (2000) and attempted to fine-tune it to establish reliable routines for measuring all the cross-anisotropic elasticity parameters of fine-grained soils by testing Japanese standard-size core specimens (i.e. a diameter of $\sim 75 \mathrm{~mm}$ ). This paper is a summary of key points from earlier works (Nishimura, 2014a and b; Martínez and Nishimura, 2015) on establishing the experimental method and applying it to a variety of natural and reconstituted fine-grained soils, ranging from clays to intermediate soils, with limited cases involving cement-stabilised clays. Some detailed discussions on parameterisation that were missed out in the earlier 
publications are also presented. An eventually proposed chart will allow 'cataloguing' patterns of stiffness anisotropy for a variety of soils in a way easy to visually perceive, leading to understanding features peculiar to each soil type.

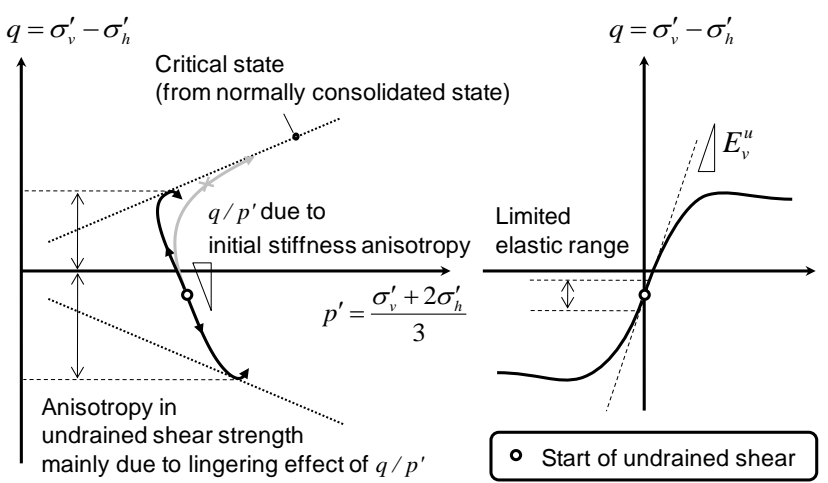

Fig. 1. Indirect effect of small-strain stiffness anisotropy on undrained strength anisotropy in brittle soils.

\section{MECHANICAL FRAMEWORK AND PARAMETERISATION}

\subsection{State-dependent cross-anisotropic elasticity}

Cross-anisotropic elasticity with state-dependent parameters is a satisfactory framework for most sedimentary and artificial (such as cement-stabilised) soils under axi-symmetric initial stress conditions. It expresses the effective stress - strain relationship as

$$
\left\{\begin{array}{l}
\delta \varepsilon_{x} \\
\delta \varepsilon_{y} \\
\delta \varepsilon_{z} \\
\delta \gamma_{x y} \\
\delta \gamma_{y z} \\
\delta \gamma_{z x}
\end{array}\right\}=\left[\begin{array}{cccccc}
1 / E_{h}^{\prime} & -v_{h h}^{\prime} / E_{h}^{\prime} & -v_{v h}^{\prime} / E_{v}^{\prime} & 0 & 0 & 0 \\
-v_{h h}^{\prime} / E_{h}^{\prime} & 1 / E_{h}^{\prime} & -v_{v h}^{\prime} / E_{v}^{\prime} & 0 & 0 & 0 \\
-v_{h v}^{\prime} / E_{h}^{\prime} & -v_{h v}^{\prime} / E_{h}^{\prime} & 1 / E_{v}^{\prime} & 0 & 0 & 0 \\
0 & 0 & 0 & 1 / G_{h h} & 0 & 0 \\
0 & 0 & 0 & 0 & 1 / G_{h v} & 0 \\
0 & 0 & 0 & 0 & 0 & 1 / G_{v h}
\end{array}\right]\left\{\begin{array}{l}
\delta \sigma_{x}^{\prime} \\
\delta \sigma_{y}^{\prime} \\
\delta \sigma_{z}^{\prime} \\
\delta \tau_{x y} \\
\delta \tau_{y z} \\
\delta \tau_{z x}
\end{array}\right\}
$$

where $E^{\prime}, G$ and $v^{\prime}$ represent Young's moduli, the shear moduli and Poisson's ratios, respectively, and the subscripts $v$ and $h$ represent the vertical (set coincident with the $z$-axis) and horizontal directions, respectively. There are three conditions that reduces number of independent parameters in the matrix to five; $G_{v h}=G_{h h}$, $v_{v h}{ }^{\prime} / E_{v}{ }^{\prime}=v_{h v}{ }^{\prime} / E_{h}{ }^{\prime}$, and $G_{h h}=E_{h}{ }^{\prime} / 2\left(1+v_{h h}\right)$. After Roesler (1979) and Hoque et al. (1996), each modulus is known to be dependent on the effective normal stresses on the planes represented by the subscripts;

$$
\begin{aligned}
& E_{v}^{\prime}=C_{v} f(e)\left(\sigma_{v}^{\prime} / p_{r}\right)^{2 m_{v}} \\
& E_{h}^{\prime}=C_{h} f(e)\left(\sigma_{h}^{\prime} / p_{r}\right)^{2 m_{h}} \\
& G_{v h}=C_{v h} f(e)\left(\sigma_{v}^{\prime} / p_{r}\right)^{n_{v}}\left(\sigma_{h}^{\prime} / p_{r}\right)^{n_{h}} \\
& G_{h h}=C_{h h} f(e)\left(\sigma_{h}^{\prime} / p_{r}\right)^{2 n_{h h}}
\end{aligned}
$$

where $C, m$ and $n$ are constants. The void ratio function, $f(e)$ takes account of the effect of state, along with the effective stress terms. In the data shown later, $f(e)=e^{-1.3}$ was adopted after Jamiolkowski et al. (1995). The reference pressure, $p_{r}$, is an arbitrarily defined constant to non-dimensionalise the expression. It is often set either as $1 \mathrm{kPa}$ or as a nominal atmospheric pressure $(101.3 \mathrm{kPa}$ or $100 \mathrm{kPa})$. The constant $C$ 's essentially represent moduli at $f(e)=1$ and $\sigma_{v}{ }^{\prime}=\sigma_{h}{ }^{\prime}=p_{r}$. The difference between $C_{v}, C_{h}, C_{v h}$ and $C_{h h}$ reflect inherent anisotropy.

The constants are easily obtained by applying linear regression in log-log scales to values of each modulus normalised by $f(e)$ measured at multiple isotropic or anisotropic stress states, as illustrated in Fig. 2. When constants $C$ 's are compared between different soils tested under widely different stress levels, a caution is necessary. Unless they share the reference pressure, $p_{r}$, within or close to their tested stress ranges, the derived $C$ values reflect unintended extrapolation, as seen in Fig. 2. A small divergence of modulus $/ f(e)-$ stress product relationships, much subject to experimental errors, will be unduly amplified. This fact suggests that a universally consistent definition of $C$ based on a single $p_{r}$ value may be difficult to establish, as natural soils exist in a variety of stress ranges. When cataloguing stiffness anisotropy patterns seen for a variety of soils, a different approach is necessary. This study focuses only on the ratios among four constants $C_{v}, C_{h}, C_{v h}$ and $C_{h h}$ as measure of inherent anisotropy. Determining and comparing such ratios between different soils will not require setting a common $p_{r}$ value.

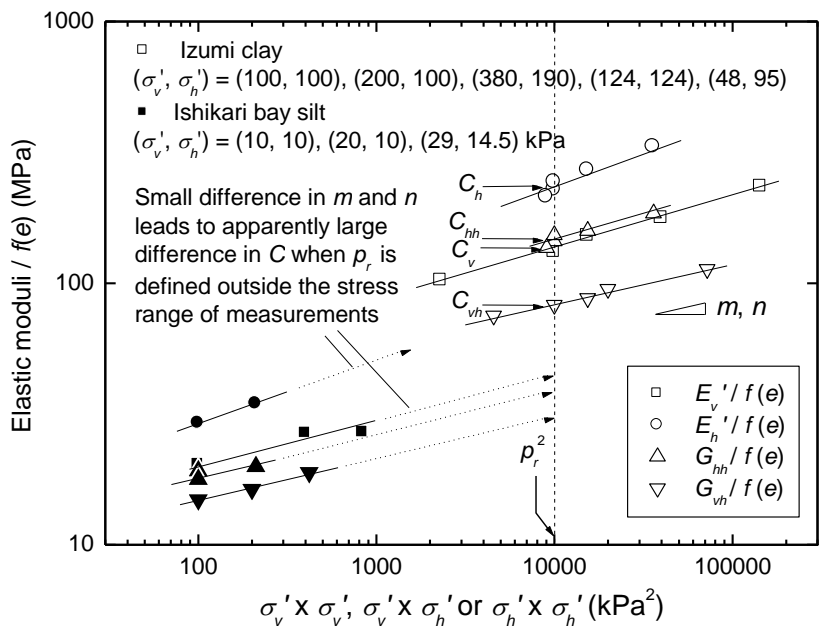

Fig. 2. Determination of constants via linear regression in log-log plots and cautionary note on defining $p_{r}$.

\subsection{Inherent anisotropy indices}

Given the five degrees of freedom (DOFs) present in the cross-anisotropic elasticity parameters, four ratios will sufficiently describe stiffness anisotropy, with one discarded DOF representing the absolute magnitude of the stiffness. These four ratios are to be defined with $C_{v}$, $C_{h}, C_{v h}$ and $C_{h h}$ plus Poisson's ratios. While a variety of 
ratios can be devised, Nishimura (2014b) proposed the following ratios as anisotropy indices;

$$
\begin{aligned}
& a_{1}=C_{h} / C_{v} \\
& a_{2}=C_{h h} / C_{v h} \\
& a_{3}=2\left(1+v_{v h}^{\prime}\right) C_{v h} / C_{v} \\
& a_{4}=3 C_{v h} / C_{v}^{u}
\end{aligned}
$$

where

$$
C_{v}^{u}=\frac{C_{v}\left[2\left(1-v_{h h}^{\prime}\right) C_{v}+\left(1-4 v_{v h}^{\prime}\right) C_{h}\right]}{2\left[\left(1-v_{h h}^{\prime}\right) C_{v}-2 v_{v h}^{\prime 2} C_{h}\right]}
$$

Ratios $a_{1}$ and $a_{2}$ correspond to commonly used ratios of $E_{h}{ }^{\prime} / E_{v}{ }^{\prime}$ and $G_{v h} / G_{h h}$, respectively, at isotropic stress states. The above four ratios are chosen so that they all become 1 when isotropy holds. The constant $C_{v}{ }^{u}$ corresponds to undrained vertical Young's modulus, $E_{v}{ }^{u}$, calculated as Eq.(10) from the effective stress-based parameters by assuming that undrained conditions are equal to constant-volume conditions. For isotropic elasticity, $3 G / E_{v}{ }^{u}=1$ is a well known relationship. The above set of four ratios is independent and retains complete information bar the magnitude of $C$ 's. In other words, if any single value for either of $C_{v}$, $C_{h}, C_{v h}$ or $C_{h h}$ is given, $a_{1}-a_{4}$ recover all of them. This inverse course follows the steps below. Firstly, $C_{v h} / C_{v}$ is obtained as a solution of the following parabolic equation;

$$
\begin{aligned}
12\left(a_{2}-a_{1} a_{2}\right)\left(\frac{C_{v h}}{C_{v}}\right)^{2}- & \left(3 a_{1}-12 a_{1} a_{2} a_{3}+4 a_{2} a_{4}+5 a_{1} a_{2} a_{4}\right) \frac{C_{v h}}{C_{v}} \\
& +2 a_{1} a_{2} a_{3} a_{4}+a_{1} a_{4}-3 a_{1} a_{2} a_{3}^{2}=0
\end{aligned}
$$

Poisson's ratio, $v_{v h}$ ', is then given as

$$
v_{v h}^{\prime}=\frac{a_{3}}{2} \frac{C_{v h}}{C_{v}}-1
$$

Once $v_{v h}{ }^{\prime}$ is obtained, any single value of $C_{v}, C_{h}, C_{v h}$ or $C_{h h}$ will lead to all the rest straightforwardly via $a_{1}-a_{4}$. A radar chart defined by $a_{1}-a_{4}$ visualises the impression of anisotropy pattern well, as demonstrated later.

\section{EXPERIMENTAL METHOD: KEY ISSUES}

\subsection{Methodology and apparatus}

Determining the constants in Eqs.(2)-(5) requires measuring the elastic parameters at multiple stress points. At each point, a set of drained small-strain probe loadings, involving axial loadings with constant radial stress and radial loadings with constant axial stress, was performed with direct-drive motor-driven triaxial apparatus with full local instrumentation. Following Kuwano et al. (2000) and Lings et al. (2000), the above static probes were couple with multi-directional shear wave velocity probes with vertical and horizontal pairs of bender elements. An example of apparatus is shown in Fig. 3, and detailed requirements for instrumentation are discussed in Nishimura (2014a). The key issues among others are;

(i) All the local displacement transducers must be capable of resolving the axial and radial strains to order of $10^{-4} \%$.

(ii) For obtaining the elastic parameters for clays, the stress-strain control and measurement must be stable over hours, as the loading rate to be adopted is extremely slow (typically $0.001 \%$ /hour).

(iii) The shear wave velocity measurement by bender elements must be calibrated well in advance, preferably through parallel static measurements of $G_{v h}$ in hollow cylinder apparatus. Although past studies on bender element interpretation (e.g. Viggiani and Atkinson, 1995; Jovičić et al., 1996; Yamashita et al., 2009) attempted reducing subjectivity in interpretation, no universal way of interpretation has been devised to guarantee the trueness of output $G$ values. A direct calibration through parallel tests, even on a limited number of samples, leads to increased confidence in $G_{v h}$, and to some degree, $G_{h h}$ determination.

(iv) A due care must be taken to minimise any ambient temperature fluctuation, preferably to less than $+/-0.2^{\circ} \mathrm{C}$ (this value may depend on the cell size; a greater cell water volume will buffer the effect) to observe consistency in the static measurement.
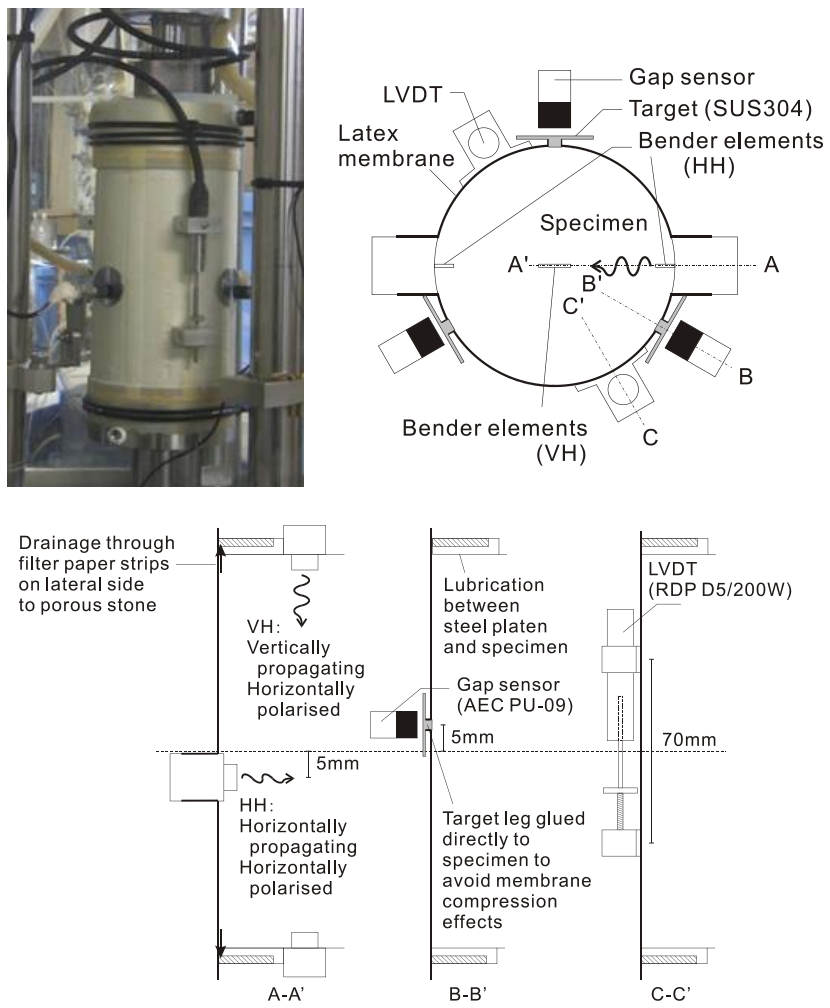

Fig. 3. Multi-instrumented triaxial apparatus adopted in this study.

3.2 Proposed procedures and modulus derivation The original method proposed by Kuwano et al. 
(2000) and Lings et al. (2000) uses the static axial and radial probes, with both axial and radial responses always measured in each of them, combined with dynamically measured $G_{v h}$ and $G_{h h}$. Nishimura (2014a) proposed an alternative method, in which additional quick static undrained probe in the axial direction is additionally conducted. At the expense of this limited effort (undrained loadings can be conducted much more quickly and readily than the drained ones), this method removes the need for radial displacement measurement in determining all the cross-elastic parameters. More detailed discussions and the adopted equations are reported by Nishimura (2014a). Fig. 4 compares the drained horizontal Young's modulus obtained with and without the radial displacement sensors, calculated via different derivation routes, for six Japanese clays reported by Nishimura (2014b). As the alternative method involves an assumption that undrained conditions are constant-volume conditions, they diverge slightly when stiff soils are tested. However, the agreement is mostly satisfactory.

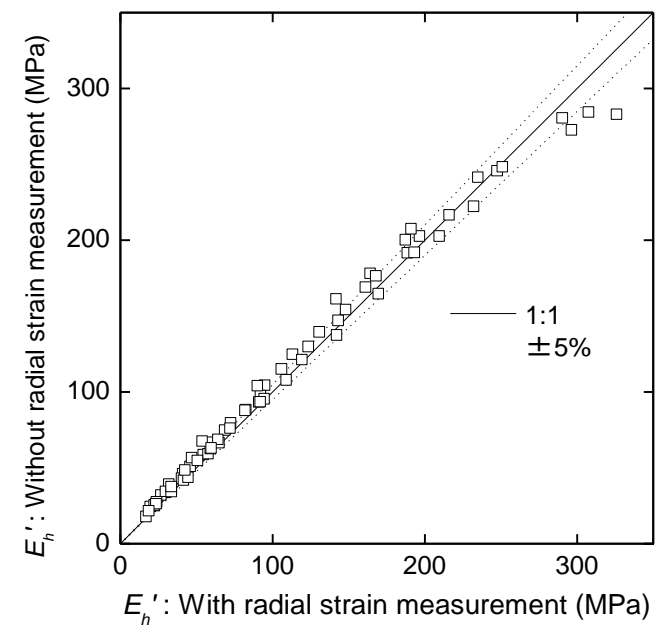

Fig. 4. Comparison of drained horizontal Young's modulus, $E_{h}{ }^{\prime}$, calculated by different methods.

\section{EXAMPLES OF INHERENT STIFFNESS ANISOTROPY EXPRESSED IN FULL}

\subsection{Inherent anisotropy chart}

The structure of the model expressed by Eqs.(2)-(5) is clear; the stress-induced anisotropy is expressed by the exponential terms, and the rest represent the inherent anisotropy. Existing studies (e.g. Jamiolkowski et al., 1995) and the first author's experience indicate that the values of the exponents $m$ and $n$ are broadly similar for all the moduli. The assessment of anisotropy then simply comes down to understanding the patterns of the constant $C$. This can be done by observing a chart defined by $a_{1}-a_{4}$ introduced earlier. Fig. 5 shows the ranges observed for natural and reconstituted states of six Japanese clays. Individual charts for each clay revealed that theese two states share striking similarity in the inherent anisotropy patterns, provided that they are both $\mathrm{K}_{0}$ consolidated (Nishimura, 2014b). $\mathrm{K}_{0^{-}}$ consolidated clays' stiffness anisotropy is characterised by very large $a_{1}$ and moderately large $a_{2}$ and $a_{3}$, all in excess of 1 , and $a_{4}$ close to 1 . The observation $a_{4} \approx 1$ indicates that $E_{v}{ }_{v} \approx 3 G_{v h}$ at isotropic stress states. As discussed in the introduction, this observation, readily made in laboratory with relatively basic instrumentation (i.e. axial strain sensors and a single vertical bender element pair) gives a misleading impression of isotropy, while more advanced characterisation showing $a_{1}-a_{3}>1$ would reveal otherwise.

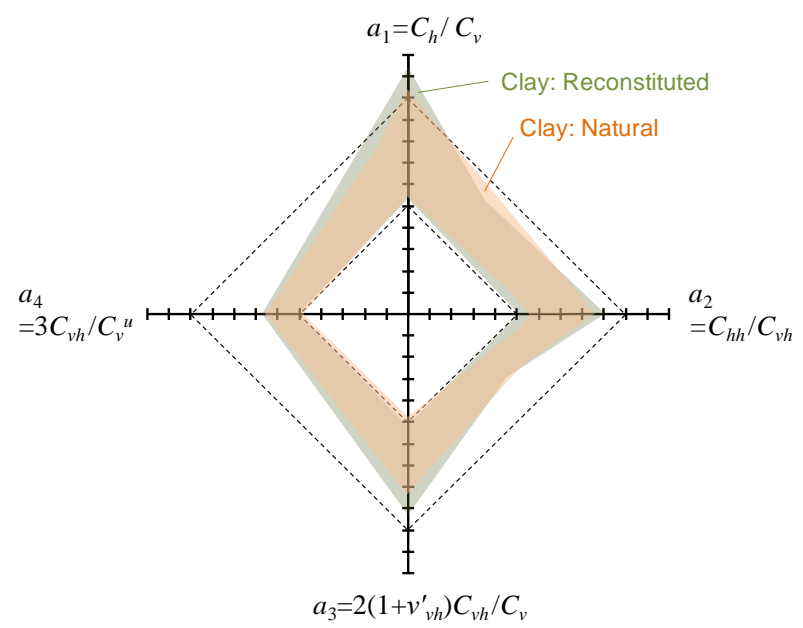

Fig. 5. Range of inherent anisotropy patterns represented by $a_{1}-a_{4}$ for natural and reconstituted clays (summary plot of data from Nishimura, 2014b).

\subsection{Anisotropy patterns of different soils}

A chart in form of Fig. 5 provides a platform on which inherent anisotropy of different soils are catalogued and compared, although the published data of complete cross-anisotropic parameter sets are still limited in number. This section reviews some of them; most of them were obtained from triaxial tests performed similarly as presented earlier, but the data on sands include those from large-size calibration chamber tests with multi-array geophysical instrumentation (Bellotti et al., 1996). Where the elastic parameters are available only for a single isotropic stress, $a_{1}-a_{4}$ were calculated by using $E$ and $G$, not $C$.

(i) Pluviated sands and glass ballotini

Uniform sands and glass ballotini were tested by Bellotti et al. (1996) and Kuwano and Jardine (2002). Their anisotropy patterns at isotropic stresses are shown in Fig. 6(a) and (b), respectively, against those of the clays in the background. These coarser granular materials clearly proved much more isotropic than clays. Kuwano and Jardine, quoting a discrete element modelling study, explained this by isotropic development of force chains within the particle assembly under isotropic stresses. It is well likely that the origin and nature stiffness anisotropy in $\mathrm{K}_{0}$-consolidated clays and pluviated sands is essentially different. 


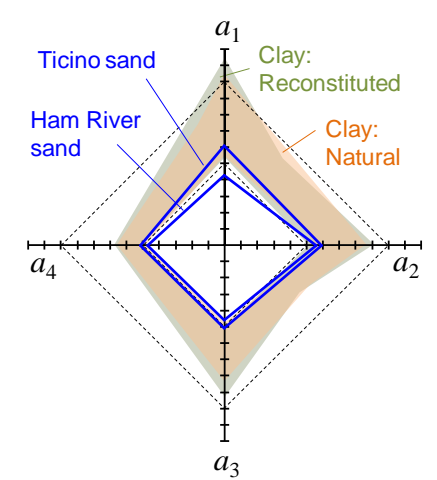

(a)

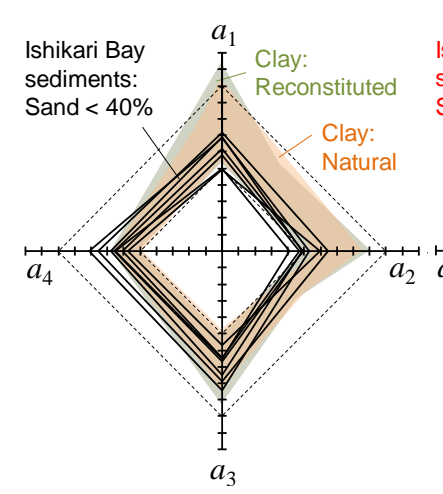

(c)

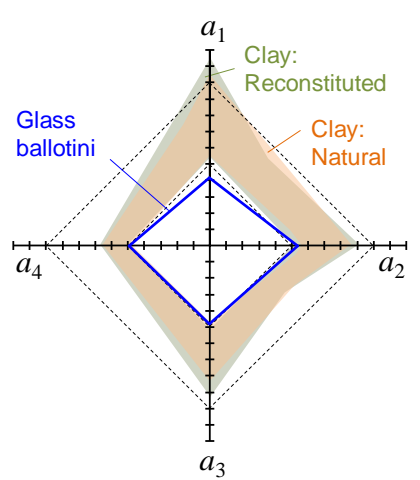

(b)

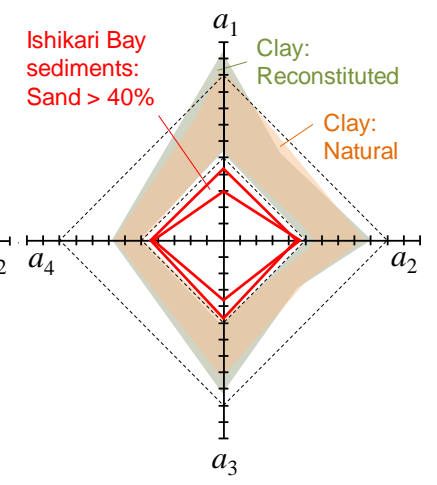

(d)
Fig. 6. Inherent anisotropy patterns for sands, glass ballotini and non-uniform Ishikari Bay sediments; (a) Ticino sand (Bellotti et al., 1996) and Ham River sand (Kuwano and Jardine, 2002); (b) Glass ballotini (Kuwano and Jardine, 2002); (c)(d) Ishikari Bay sediments (Martínez and Nishimura, 2015).

(ii) Intermediate soils: Ishikari Bay sediments

Following a similar scheme as Nishimura (2014b), Martínez and Nishimura (2015) tested Holocene and Pleistocene silty sediments from a breakwater construction site at New Ishikari Bay Port, Hokkaido, Japan. Profiles of some index properties and soil ages assessed by radiocarbon dating analysis are shown in Fig. 7. The data in this paper have been updated since the above publication. The anisotropy patterns are shown in two plots, grouped according to a threshold sand content of 40\%, in Fig. 6(c) and (d). Clearly, the stiffness anisotropy of the predominantly sandy group agrees with that of the uniform sands shown in Fig. 6(a) and (b), while the pattern became gradually 'intermediate' between that of clays and sands, as the fines content increased. Although no straight forward correlation between the index properties and the anisotropy pattern was identified, these transitional anisotropy changes seem rational.

\section{(iii) Air-foam/cement mixed clays}

It is interesting to extend the 'catalogue' to artificially prepared soils. Nishimura et al. (2016) tested air-foam/cement mixed high-water content clays (prepared in compliance with the 'SGM' technique) with four different constituent ratios, as shown in Table 1. The anisotropy patterns, shown in Fig. 8, are distinctly different from those of natural soils. Although the authors' study on these materials has not been as extensive as on natural soils and any conclusion is tentative, the specimen with the smallest volume fraction of clay particles exhibited a fairly isotropic pattern. Although Mix 1 and 4 still have small soil volume fractions, their relatively high unconfined compression strength suggests that the hydration filled larger proportions of the original voids. The patterns seen in Fig. 8 suggest that the resulting structure is not necessarily isotropic.

\section{CONCLUSIONS}

This paper looked into small-strain stiffness anisotropy of natural, reconstituted and artificially mixed/stabilised soils through parameterisation of cross-anisotropic elasticity parameters. The experimental methods and key issues involved are briefly reviewed. Appropriately determined parameters are conveniently converted into four ratios that

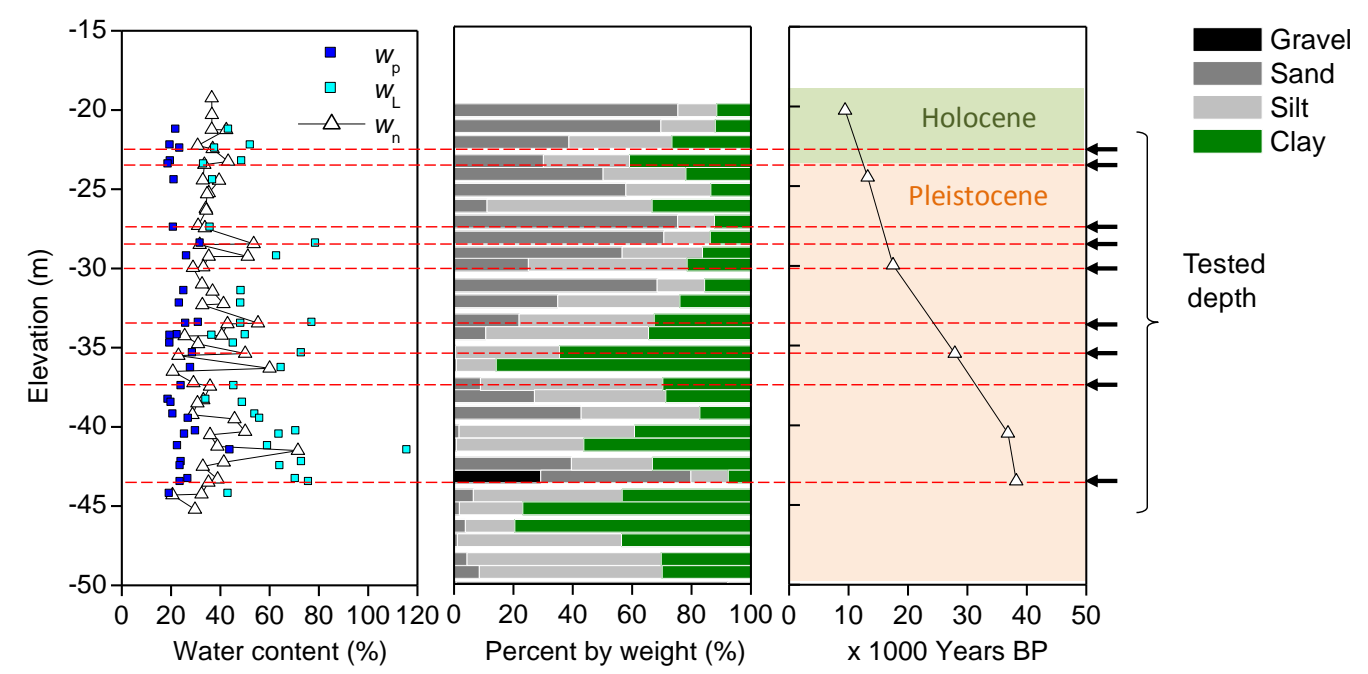

Fig. 7. Profiles of index properties and soil ages for Ishikari Bay sediments (the sea bottom at elevation of $-18.5 \mathrm{~m}$ ) Table 1. Mixing conditions of air-foam/cement mixed clays 


\begin{tabular}{|c|c|c|c|c|}
\hline Mixing conditions and properties & Mix 1 & Mix 2 & Mix 3 & Mix 4 \\
\hline$w / w_{L}$ of soil slurry $(\%)$ & 200 & 250 & 250 & 250 \\
\hline$W_{w} / W_{s}$ of soil slurry $(\%)$ & 124 & 155 & 155 & 155 \\
\hline$W_{c} / W_{s}$ of slurry-cement-air mixture $(\%)$ & 20 & 20 & 10 & 20 \\
\hline$V_{a} / W_{s}$ of slurry-cement-air mixture $(l / \mathrm{kgf})$ & 0.6 & 0.6 & 0.6 & 0 \\
\hline Volumetric fraction of soil particles & 0.16 & 0.14 & 0.15 & 0.19 \\
\hline Unconfined compression strength & 012 & 162 & 65 & 861 \\
\hline
\end{tabular}

$w$ : Water content, $w_{L}$ : Liquid limit of clay, $W_{w}$ : Weight of water, $W_{s}$ : Weight of dry soil, $W_{c}$ : Weight of cement, $V_{a}:$ Volume of air

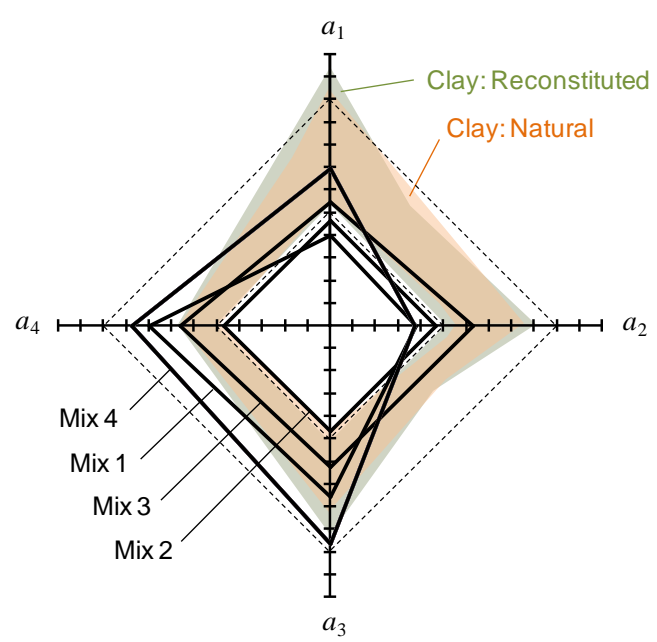

Fig. 8. Inherent anisotropy patterns for air-foam/cement mixed clays (data from Nishimura et al., 2016).

represent inherent anisotropy, or the inter-moduli ratios at isotropic stresses. Through visualisation in form of a chart, inherent stiffness anisotropy patterns were compared between different soil types, including natural and reconstituted clays, uniform sands, glass ballotini, natural non-uniform silts and air-foam/cement mixed clays. Although it is not all understood how the microstructure in each soil type gives rise to its peculiar anisotropy patterns, consistent trends were observed that seem to correlate well with gradation. The proposed scheme offers a platform for further exploration of stiffness anisotropy in various soils.

\section{ACKNOWLEDGMENTS}

The data presented in this paper were obtained in multiple projects funded by different bodies; KAKENHI Grant Number 23760436, JSPS, Ministry of Land, Infrastructure, Transport and Tourism, Japan, and NOASTEC, Japan. Their support is sincerely acknowledged.

\section{REFERENCES}

1) Addenbrooke, T. I., Potts, D. M. and Puzrin, A. M. (1997): The influence of pre-failure soil stiffness on the numerical analysis of tunnel construction, Géotechnique, 47(3), 693-712.

2) Bellotti, R., Jamiolkowski, M., Lo Presti, D. C. F. and O’Neill, D. A. (1996): Anisotropy of small strain stiffness in
Ticino sand, Géotechnique, 46(1), 115-131.

3) Graham, J. and Houlsby, G. T. (1983): Anisotropic elasticity of a natural clay, Geotechnique, 33(2), 165-180.

4) Hoque, E., Tatsuoka, F. and Sato, T. (1996): Measuring anisotropic elastic properties of and using a large triaxial specimen, Geotechnical Testing Journal, 19(4), 411-420.

5) Kuwano, R, Connolly, T. M. and Jardine, R. J. (2000): Anisotropic stiffness measurements in a stress-path triaxial cell, Geotechnical Testing Journal, 23(2), 141-157.

6) Kuwano, R. and Jardine, R. J. (2002): On the applicability of cross-anisotropic elasticity to granular materials at very small strains, Géotechnique, 52 (10), 727-749.

7) Jamiolkowski, M., Lancellotta, R. and Lo Presti, D. C. F. (1995): Remarks on the stiffness at small strains of six Italian clays, 1 st International Conference on Pre-failure Deformation Characteristics of Geomaterials, Sapporo, Japan, Vol.1 817-836.

8) Jovičić, V., Coop, M. R. and Simić, M. (1996): Objective criteria for determining $G_{\max }$ from bender element tests, Géotechnique, 46(2), 357-362.

9) Lings, M. L., Pennington, D. S. and Nash, D. F. T. (2000): Anisotropic stiffness parameters and their measurement in a stiff natural clay, Géotechnique, 50(2), 109-125.

10) Martínez, D. A. and Nishimura, S. (2015): Stiffness anisotropy characteristics of natural fined-grained seabed sediments, 6th International Symposium on Deformation Characteristics of Geomaterials, Buenos Aires, pp.824-831.

11) Nishimura, S. (2006): Laboratory study on anisotropy of natural London Clay, $\mathrm{PhD}$ Thesis, Imperial College London.

12) Nishimura, S. (2014a): Assessment of anisotropic elastic parameters of saturated clay measured in triaxial apparatus: Appraisal of techniques and derivation procedures, Soils and Foundations, 54(3), 364-376.

13) Nishimura, S. (2014b): Cross-anisotropic deformation characteristics of natural sedimentary clays, Géotechnique, 64(12), 981-996.

14) Nishimura, S., Iwaki, A., Takashino, S. and Tanaka, H. (2016): Image-based measurement of one-dimensional compressibility in cement-treated soils, Géotechnique, 66(10), 840-853.

15) Roesler, S. K. (1979): Anisotropic shear modulus due to stress anisotropy, Journal of the Geotechnical Engineering Division, ASCE 105(GT7), 871-880.

16) Simpson, B., Atkinson, J. H. and Jovičić, V. (1996): The influence of anisotropy on calculation of ground settlements above tunnels, 1st International Symposium on Geotechnical Aspects of Underground Construction in Soft Ground, London, pp.591-595.

17) Viggiani, G. and Atkinson, J. H. (1995): Interpretation of bender element test, Géotechnique, 45(1), 149-154.

18) Yamashita, S., Kawaguchi, T., Nakata, Y., Mikami, T., Fujiwara, T. and Shibuya, S. (2008): Interpretation of international parallel test on the measurement of $\mathrm{G}_{\max }$ using bender elements, Soils and Foundations, 49(4), 631-650. 